Article

\title{
On the Oscillatory Behavior of a Class of Fourth-Order Nonlinear Differential Equation
}

\author{
Osama Moaaz ${ }^{1,+} \mathbb{D}$, Poom Kumam $2,3, *,+\mathbb{D}$ and Omar Bazighifan $1,4,5,+(\mathbb{D}$ \\ 1 Department of Mathematics, Faculty of Science, Mansoura University, Mansoura 35516, Egypt; \\ o_moaaz@mans.edu.eg (O.M.); o.bazighifan@gmail.com (O.B.) \\ 2 Center of Excellence in Theoretical and Computational Science (TaCS-CoE) and Department of Mathematics, \\ Faculty of Science, King Mongkuts University of Technology Thonburi (KMUTT), 126 Pracha Uthit Rd., \\ Bang Mod, Thung Khru, Bangkok 10140, Thailand \\ 3 Department of Medical Research, China Medical University Hospital, China Medical University, \\ Taichung 40402, Taiwan \\ 4 Department of Mathematics, Faculty of Science, Hadhramout University, Hadhramout 50512, Yemen \\ 5 Department of Mathematics, Faculty of Education, Seiyun University, Hadhramout 50512, Yemen \\ * Correspondence: poom.kumam@mail.kmutt.ac.th \\ + These authors contributed equally to this work.
}

Received: 12 February 2020; Accepted: 17 March 2020; Published: 2 April 2020

\begin{abstract}
In this work, we study the oscillatory behavior of a class of fourth-order differential equations. New oscillation criteria were obtained by employing a refinement of the Riccati transformations. The new theorems complement and improve a number of results reported in the literature. An example is provided to illustrate the main results.
\end{abstract}

Keywords: Oscillatory solutions; fourth-order; delay differential equations

\section{Introduction}

In this paper, we are concerned with the oscillation and the asymptotic behavior of solutions of the fourth-order nonlinear differential equation

$$
\left(r(t)\left(x^{\prime \prime \prime}(t)\right)^{\alpha}\right)^{\prime}+q(t) x^{\beta}(\sigma(t))=0,
$$

where $\alpha$ and $\beta$ are quotient of odd positive integers, $r \in C^{1}\left(\left[t_{0}, \infty\right)\right), q \in C\left(\left[t_{0}, \infty\right)\right), r(t)>0$, $q(t)>0, r^{\prime}(t) \geq 0, \sigma(t) \in C\left(\left[t_{0}, \infty\right), \mathbb{R}\right), \sigma(t) \leq t, \lim _{t \rightarrow \infty} \sigma(t)=\infty$. Moreover, we study (1) under the condition

$$
\int_{t_{0}}^{\infty} \frac{1}{r^{1 / \alpha}(s)} \mathrm{d} s=\infty
$$

We intend to a solution of (1) a function $x(t):\left[t_{x}, \infty\right) \rightarrow \mathbb{R}, t_{x} \geq t_{0}$ such that $x(t)$ and $r(t)\left(x^{\prime \prime \prime}(t)\right)^{\alpha}$ are continuously differentiable for all $t \in\left[t_{x}, \infty\right)$ and $\sup \{|x(t)|: t \geq T\}>0$ for any $T \geq t_{x}$. We assume that (1) possesses such a solution. A solution $y$ is said to be non-oscillatory if it is eventually positive or eventually negative; otherwise, it is said to be oscillatory. (1) is said to be oscillatory if all its solutions are oscillatory. The equation itself is called oscillatory if all of its solutions are oscillatory.

The reliance on the past shows up normally in various applications in biology, electrical engineering or physiology. A basic model in nature is reforestation. A cut timberland, in the wake of replanting, will take in any event 20 years before arriving at any sort of development. Consequently, any scientific model of backwoods gathering and recovery plainly should have time defers incorporated with it. Another model happens because of the way that creatures must set aside some effort to process 
their nourishment before further exercises and reactions occur. Consequently, any model of species dynamics without delays is an approximation at best, see [1].

For several decades, an growing interest in studying the oscillation and non-oscillation criteria of different classes and different orders of differential equations with delay has been observed; see, for instance, the monographs [2,3], the papers [4-26], and the references cited therein.

The purpose of this paper is to give new sufficient conditions for the oscillatory behavior of (1). In Section 2, we will provide some auxiliary lemmas that will help us to prove our oscillation criteria. In Section 3, by employing a refinement of the Riccati transformations, we establish new oscillation criteria of (1).

\section{Auxiliary Lemmas}

Notation 1. Here, we introduce Riccati substitutions

$$
\omega_{1}(t):=\frac{r(t)\left(x^{\prime \prime \prime}(t)\right)^{\alpha}}{x^{\alpha}(t)}
$$

and

$$
\omega_{2}(t):=\frac{x^{\prime}(t)}{x(t)}
$$

Moreover, for convenience, we denote that

$$
Q(t):=M_{1}^{\beta-\alpha} q(t) \frac{\sigma^{3 \alpha}(t)}{t^{3 \alpha}}, R_{1}(t):=\frac{\alpha \mu}{2} \frac{t^{2}}{r^{1 / \alpha}(t)},
$$

and

$$
\widetilde{R}(t):=\lambda^{\beta / \alpha} M_{2}^{\beta-\alpha} \int_{t}^{\infty}\left(\frac{1}{r(u)} \int_{u}^{\infty} q(s)\left(\frac{\sigma(s)}{s}\right)^{\beta} \mathrm{d} s\right)^{1 / \alpha} \mathrm{d} u,
$$

where $\mu, \lambda \in(0,1)$ and $M_{1}, M_{2}$ are positive constants.

All functional inequalities are assumed to hold eventually, that is, they are assumed to be satisfied for all $t$ sufficiently large. We begin with the following lemmas that can be found in $[2,8,16,18]$, respectively.

Lemma 1. Let $h \in C^{n}\left(\left[t_{0}, \infty\right)\right)$ and $h(t)>0$. Suppose that $h^{(n)}(t)$ is of a fixed sign, on $\left[t_{0}, \infty\right), h^{(n)}(t)$ not identically zero and that there exists $a t_{1} \geq t_{0}$ such that, for all $t \geq t_{1}$,

$$
h^{(n-1)}(t) h^{(n)}(t) \leq 0
$$

If we have $\lim _{t \rightarrow \infty} h(t) \neq 0$, then there exists $t_{\lambda} \geq t_{0}$ such that

$$
h(t) \geq \frac{\lambda}{(n-1) !} t^{n-1}\left|h^{(n-1)}(t)\right|,
$$

for every $\lambda \in(0,1)$ and $t \geq t_{\lambda}$.

Lemma 2. If the function $x$ satisfies $x^{(i)}(t)>0, i=0,1, \ldots, n$, and $x^{(n+1)}(t)<0$, then

$$
\frac{x(t)}{t^{n} / n !} \geq \frac{x^{\prime}(t)}{t^{n-1} /(n-1) !} \text {. }
$$


Lemma 3. Assume that $\alpha$ is a quotient of odd positive integers. Then

$$
U y-V y^{(\alpha+1) / \alpha} \leq \frac{\alpha^{\alpha}}{(\alpha+1)^{\alpha+1}} U^{\alpha+1} V^{-\alpha}, V>0 .
$$

Lemma 4. Assume that (2) is satisfied and let $x(t)$ be an eventually positive solution of (1). Then, there exists a sufficiently large $t_{1} \geq t_{0}$ such that for all $t \geq t_{1}$, either

$$
\left(S_{1}\right) x^{(\kappa)}(t)>0 \text { for } \kappa=0,1,2,3 \text {; }
$$

or

$$
\left(S_{2}\right) x^{(\kappa)}(t)>0 \text { for } \kappa=0,1,3 \text {, and } x^{\prime \prime}(t)<0 \text {, }
$$

is holds.

Lemma 5. Let $x(t)$ is an eventually positive solution of Equation (1).

(i $\left.\mathbf{i}_{1}\right)$ If $x$ satisfies $\left(S_{1}\right)$, then

$$
\omega_{1}^{\prime}(t)+Q(t)+R_{1}(t) \omega_{1}^{1+1 / \alpha}(t) \leq 0 ;
$$

(i $\left.\mathbf{i}_{2}\right)$ If $x$ satisfies $\left(S_{2}\right)$, then

$$
\omega_{2}^{\prime}(t)+\omega_{2}^{2}(t)+B^{\beta-\alpha} \widetilde{R}(t) \leq 0 .
$$

Proof. Let that $x(t)$ is an eventually positive solution of Equation (1). From Lemmas 4, there exist two possible cases $\left(S_{1}\right)$ and $\left(S_{2}\right)$ for $t \geq t_{1}$ large enough.

Let $\left(S_{1}\right)$ holds. Then, taking Lemma 1 and 2 into account, we arrive at

$$
x^{\prime}(t) \geq \frac{\mu}{2} t^{2} x^{\prime \prime \prime}(t)
$$

and $x(t) \geq \frac{1}{3} t x^{\prime}(t)$. Hence,

$$
x(\sigma(t)) \geq \frac{\sigma^{3}(t)}{t^{3}} x(t) .
$$

Differentiating $\omega_{1}$ and using (1), (6) and (7), we obtain

$$
\omega_{1}^{\prime}(t) \leq-q(t) \frac{\sigma^{3 \alpha}(t)}{t^{3 \alpha}} x^{\beta-\alpha}(\sigma(t))-\frac{\alpha \mu}{2} \frac{t^{2}}{r^{1 / \alpha}(t)} \omega_{1}^{1+1 / \alpha}(t) .
$$

Since $x^{\prime}(t)>0$, there exist a $t_{2} \geq t_{1}$ and a constant $B>0$ such that $x(t)>B$, for all $t \geq t_{2}$. Thus, we see that

$$
\omega_{1}^{\prime}(t) \leq-q(t) \frac{\sigma^{3 \alpha}(t)}{t^{3 \alpha}} B^{\beta-\alpha}(\sigma(t))-\frac{\alpha \mu}{2} \frac{t^{2}}{r^{1 / \alpha}(t)} \omega_{1}^{1+1 / \alpha}(t),
$$

Thus, (4) is satisfied.

Let $\left(S_{2}\right)$ holds. Integrating (1) from $t$ to $l$, we have

$$
r(l)\left(x^{\prime \prime \prime}(l)\right)^{\alpha}=r(t)\left(x^{\prime \prime \prime}(t)\right)^{\alpha}-\int_{t}^{l} q(s) x^{\beta}(\sigma(s)) \mathrm{d} s .
$$

Taking Lemma 2 into account, we arrive at

$$
x(t) \geq t x^{\prime}(t) .
$$

Thus, $x(\sigma(t)) \geq(\sigma(t) / t) x(t)$, which with (8) and the fact that $x^{\prime}(t)>0$ gives

$$
r(l)\left(x^{\prime \prime \prime}(l)\right)^{\alpha}-r(t)\left(x^{\prime \prime \prime}(t)\right)^{\alpha}+x^{\beta}(t) \int_{t}^{l} q(s)\left(\frac{\sigma(s)}{s}\right)^{\beta} \mathrm{d} s \leq 0 .
$$


Letting $l \rightarrow \infty$, we obtain

$$
x^{\prime \prime \prime}(t) \geq \frac{\lambda^{\beta / \alpha}}{r^{1 / \alpha}(t)} x^{\beta / \alpha}(t)\left(\int_{t}^{\infty} q(s)\left(\frac{\sigma(s)}{s}\right)^{\beta} \mathrm{d} s\right)^{1 / \alpha} .
$$

Integrating the above inequality from $t$ to $\infty$, we obtain

$$
\begin{aligned}
x^{\prime \prime}(t) & \leq-\lambda^{\beta / \alpha} x^{\beta / \alpha}(t) \int_{t}^{\infty}\left(\frac{1}{r(u)} \int_{u}^{\infty} q(s)\left(\frac{\sigma(s)}{s}\right)^{\beta} \mathrm{d} s\right)^{1 / \alpha} \mathrm{d} u \\
& \leq-\widetilde{R}(t) x^{\beta / \alpha}(t) .
\end{aligned}
$$

Differentiating $\omega_{2}$ and using (10), we get

$$
\omega_{2}^{\prime}(t)+\omega_{2}^{2}(t)+B^{\beta-\alpha} \widetilde{R}(t) \leq 0 .
$$

Thus, the proof is complete.

\section{Oscillation Criteria}

Theorem 1. If

$$
\int_{t_{0}}^{\infty} Q(s) \mathrm{d} s=\infty
$$

and

$$
\int_{t_{0}}^{\infty} \widetilde{R}(s) \mathrm{d} s=\infty
$$

then (1) is oscillatory.

Proof. Assume to the contrary that (1) has a nonoscillatory solution in $\left[t_{0}, \infty\right)$. Without loss of generality, we can assume that $x(t)>0$. From Lemma 4 that there exist two possible cases for $t \geq t_{1}$, where $t_{1} \geq t_{0}$ is sufficiently large.

For case $\left(S_{1}\right)$, from Lemma 5 , we see that (4) holds, which yields

$$
\omega_{1}^{\prime}(t)+Q(t) \leq 0
$$

Integrating (13) from $t_{2}$ to $t$ and using (11),we obtain

$$
\omega_{1}(t) \leq \omega_{1}\left(t_{2}\right)-\int_{t_{2}}^{t} Q(s) d s \rightarrow-\infty \text { as } t \rightarrow \infty,
$$

which contradicts the fact that $\omega_{1}(t)>0$.

Similarly, in the case where $\left(S_{2}\right)$ holds, we get a contradicts with (12), which is omitted here for convenience. Therefore, the proof is complete.

Definition 1. The sequence of functions $\left\{y_{n}(t)\right\}_{n=0}^{\infty}$ and $\left\{z_{n}(t)\right\}_{n=0}^{\infty}$ define as

$$
y_{n}(t)=y_{0}(t)+\int_{t}^{\infty} R_{1}(s) y_{n-1}^{\frac{\alpha+1}{\alpha}}(s) \mathrm{d} s
$$

and

$$
z_{n}(t)=z_{0}(t)+\int_{t}^{\infty} z_{n-1}^{2}(s) \mathrm{d} s,
$$

where

$$
y_{0}(t)=\int_{t}^{\infty} Q(s) \mathrm{d} s
$$


and

$$
z_{0}(t)=\int_{t}^{\infty} \widetilde{R}(s) \mathrm{d} s
$$

Theorem 2. Assume that

$$
\liminf _{t \rightarrow \infty} \frac{1}{y_{0}(t)} \int_{t}^{\infty} R_{1}(s) y_{0}^{\frac{\alpha+1}{\alpha}}(s) \mathrm{d} s>\frac{\alpha}{(\alpha+1)^{\frac{\alpha+1}{\alpha}}}
$$

and

$$
\liminf _{t \rightarrow \infty} \frac{1}{z_{0}(t)} \int_{t}^{\infty} z_{0}^{2}(s) \mathrm{d} s>\frac{1}{4}
$$

Then, (1) is oscillatory.

Proof. Assume to the contrary that (1) has a nonoscillatory solution in $\left[t_{0}, \infty\right)$. Without loss of generality, we can assume that $x(t)>0$. From Lemma 4 that there exist two possible cases for $t \geq t_{1}$, where $t_{1} \geq t_{0}$ is sufficiently large.

Let case $\left(S_{1}\right)$ holds. By using Lemma 5, we obtain (4). Integrating (4) from $t$ to $l$, we get

$$
\omega_{1}(l)-\omega_{1}(t)+\int_{t}^{l} Q(s) \mathrm{d} s+\int_{t}^{l} R_{1}(s) \omega_{2}^{\frac{\alpha+1}{\alpha}}(s) \mathrm{d} s \leq 0 .
$$

From (18), it is obvious that

$$
\omega_{1}(l)-\omega_{1}(t)+\int_{t}^{l} R_{1}(s) \omega_{1}(s) \mathrm{d} s \leq 0 .
$$

Then we conclude from (19) that

$$
\int_{t}^{\infty} R_{1}(s) \omega_{1}(s) \mathrm{d} s<\infty, \text { for } t \geq T,
$$

otherwise,

$$
\omega_{1}(l) \leq \omega_{1}(t)-\int_{t}^{l} R_{1}(s) \omega_{1}(s) \mathrm{d} s \rightarrow-\infty \text { as } l \rightarrow \infty,
$$

which contradicts to the fact that $\omega_{1}(t)>0$. Since $\omega_{1}(t)$ is positive and decreasing $\lim _{t \rightarrow \infty} \omega_{1}(t)=$ $k \geq 0$. By virtue of (20), we have $k=0$. Thus, from (18), we have

$$
\omega_{1}(t) \geq \widetilde{Q}(t)+\int_{t}^{\infty} R_{1}(s) \omega_{1}(s) \mathrm{d} s=y_{0}(t)+\int_{t}^{\infty} R_{1}(s) \omega_{1}(s) \mathrm{d} s .
$$

From (21), we have

$$
\frac{\omega_{1}(t)}{y_{0}(t)} \geq 1+\frac{1}{y_{0}(t)} \int_{t}^{\infty} R_{1}(s) y_{0}^{\frac{\alpha+1}{\alpha}}(s)\left(\frac{\omega_{1}(s)}{y_{0}(s)}\right)^{\frac{\alpha+1}{\alpha}} \mathrm{d} s, t \geq T .
$$

If we set $\delta=\inf _{t \geq T} \omega_{1}(t) / y_{0}(t)$, then obviously $\delta \geq 1$. Hence, from (16) and (22) we see that

$$
\delta \geq 1+\alpha\left(\frac{\delta}{\alpha+1}\right)^{(\alpha+1) / \alpha}
$$

or

$$
\frac{\delta}{\alpha+1} \geq \frac{1}{\alpha+1}+\frac{\alpha}{\alpha+1}\left(\frac{\delta}{\alpha+1}\right)^{(\alpha+1) / \alpha}
$$

which contradicts the admissible value of $\delta$ and $\alpha$. 
Similarly, in case $\left(S_{2}\right)$, if we set $\delta_{1}=\inf _{t \geq T_{1}} \omega(t) / z_{0}(t)$ and taking (17) into account, then we arrive at a contradicts with the admissible value of $\delta_{1}$. Therefore, the proof is complete.

Theorem 3. Assume that there exist some $y_{n}$ and $z_{n}$ such that

$$
\limsup _{t \rightarrow \infty} y_{n}(t)\left(\frac{\mu}{2} t^{2} \int_{t_{0}}^{t} r^{-1 / \alpha}(s) \mathrm{d} s\right)^{\alpha}>1
$$

and

$$
\limsup _{t \rightarrow \infty} t z_{n}(t)>1
$$

hold. Then (1) is oscillatory.

Proof. Assume to the contrary that (1) has a nonoscillatory solution in $\left[t_{0}, \infty\right)$. Without loss of generality, we can assume that $x(t)>0$. From Lemma 4 that there exist two possible cases for $t \geq t_{1}$, where $t_{1} \geq t_{0}$ is sufficiently large.

Let case $\left(S_{1}\right)$ holds. Taking Lemma 1 into account, we arrive at

$$
x(t) \geq \frac{\mu}{6} t^{3} x^{\prime \prime \prime}(t) .
$$

From the definition of $\omega$ and (25), we have

$$
\begin{aligned}
\frac{1}{\omega_{1}(t)} & =\frac{1}{r(t)}\left(\frac{x(t)}{x^{\prime \prime \prime}(t)}\right)^{\alpha} \\
& \geq \frac{1}{r(t)}\left(\frac{\mu}{6} t^{3}\right)^{\alpha}
\end{aligned}
$$

Thus,

$$
\omega_{1}(t) \frac{1}{r(t)}\left(\frac{\mu}{6} t^{3}\right)^{\alpha} \leq 1
$$

and

$$
\limsup _{t \rightarrow \infty} \omega_{1}(t)\left(\frac{\mu t^{3}}{6 r^{1 / \alpha}(t)}\right)^{\alpha} \leq 1
$$

which contradicts (23).

Similarly, in case $\left(S_{2}\right)$, we arrive at a contradicts with (24). Therefore, the proof is complete.

Corollary 1. If there exist $y_{n}$ and $z_{n}$ such that

$$
\int_{T}^{t} Q(s) \exp \left(\int_{T}^{s} R_{1}(u) y_{n}^{1 / \alpha}(u) \mathrm{d} u\right) \mathrm{d} s=\infty
$$

and

$$
\int_{T}^{t} \widetilde{R}(s) \exp \left(\int_{T}^{s} z_{n}(u) \mathrm{d} u\right) \mathrm{d} s=\infty,
$$

hold, then (1) is oscillatory.

Proof. Assume to the contrary that (1) has a nonoscillatory solution in $\left[t_{0}, \infty\right)$. Without loss of generality, we can assume that $x(t)>0$. From Lemma 4 that there exist two possible cases for $t \geq t_{1}$, where $t_{1} \geq t_{0}$ is sufficiently large.

Let case $\left(S_{1}\right)$ hold. Proceeding as in the proof of Theorem 2, we obtain (21). From (21), we have

$$
\omega_{1}(t) \geq y_{0}(t) .
$$


Moreover, by induction we can also see that $\omega_{1}(t) \geq y_{n}(t)$ for $t \geq t_{0}, n=1,2,3, \ldots$. Thus, since the sequence $\left\{y_{n}(t)\right\}_{0}^{\infty}$ monotone increasing and bounded above, it converges to $y(t)$. Letting $n \rightarrow \infty$ in (14) and using Lebesgues monotone convergence theorem, we obtain

$$
y(t)=y_{0}(t)+\int_{t}^{\infty} R_{1}(s) y^{\frac{\alpha+1}{\alpha}}(s) \mathrm{d} s
$$

From (29), we have that

$$
y^{\prime}(t)=-R_{1}(t) y^{\frac{\alpha+1}{\alpha}}(t)-Q(t) .
$$

Since $y_{n}(t) \leq y(t)$, it follows from (30) that

$$
y^{\prime}(t) \leq-R_{1}(t) y_{n}^{1 / \alpha}(t) y(t)-Q(t)
$$

Hence, we get

$$
y(t) \leq \exp \left(-\int_{T}^{t} R_{1}(s) y_{n}^{1 / \alpha}(s) \mathrm{d} s\right)\left(y(T)-\int_{T}^{t} Q(s) \exp \left(\int_{T}^{s} R_{1}(u) y_{n}^{1 / \alpha}(u) \mathrm{d} u\right) \mathrm{d} s\right) .
$$

The above inequality follows

$$
\int_{T}^{t} Q(s) \exp \left(\int_{T}^{s} R_{1}(u) y_{n}^{1 / \alpha}(u) \mathrm{d} u\right) \mathrm{d} s \leq y(T)<\infty,
$$

which contradicts (27).

Similarly, in case $\left(S_{2}\right)$, we arrive at a contradiction with (28). Therefore, the proof is complete.

Example 1. Consider the equation

$$
x^{(4)}(t)+\frac{q_{0}}{t^{4}} x\left(\frac{1}{2} t\right)=0,
$$

where $q_{0}>0$. We note that $\alpha=\beta=1, r(t)=1, \sigma(t)=t / 2$ and $q(t)=q_{0} / t^{4}$. Hence, it is easy to see that

$$
y_{0}=\frac{q_{0}}{24 t}
$$

and

$$
z_{0}(t)=\frac{q_{0}}{2 t}
$$

Thus, by using Theorem 2, Equation (31) is oscillatory if $q_{0}>36$. However, we note that $\int^{\infty} Q(s) \mathrm{d} s \neq \infty$, and hence, Theorem 1 fails.

Remark 1. Theorem 1 introduces a criterion in traditional form $\int^{\infty}(\cdot) \mathrm{d} s=\infty$. Howeover, Theorem 2 provides a better criterion which can be applied to different equations. While, we can use Theorem 3 if Theorem 2 fails.

Remark 2. Agarwal et al. [27] studied the oscillation properties of the higher-order equation

$$
\left(\left|x^{(n-1)}(t)\right|^{\alpha-1} x^{(n-1)}(t)\right)^{\prime}+q(t) f(x(\sigma(t)))=0,
$$

under the condition (2). From Theorem 2.1 in [27], Equation (31) is oscillatory if $q_{0}>96$. Thus, our results improve the results in [27].

\section{Conclusions}

New criteria for oscillation of fourth-order delay differential equations are established. By employing a refinement of the Riccati transformations, we obtain new oscillation criteria that 
improve some related results and can be used in cases where known theorems fail to apply. By applying our results to an example, we show that our results improved the results in [27]. Furthermore, in future work, we can try to study the oscillation properties of the neutral case by the same approach as that used in this work.

Author Contributions: The authors claim to have contributed equally and significantly in this paper. All authors have read and agreed to the published version of the manuscript.

Funding: The authors received no direct funding for this work.

Acknowledgments: The author thanks the reviewers for for their useful comments, which led to the improvement of the content of the paper.

Conflicts of Interest: There are no competing interests between the authors.

\section{References}

1. Hale, J.K. Theory of Functional Differential Equations; Springer: New York, NY, USA, 1977.

2. Agarwal, R.; Grace, S.; O’Regan, D. Oscillation Theory for Difference and Functional Differential Equations; Springer Science \& Business Media: Berlin, Germany, 2000.

3. Gyori, I.; Ladas, G. Oscillation Theory of Delay Differential Equations with Applications; Clarendon Press: Oxford, UK, 1991.

4. Bazighifan, O.; Cesarano, C. Some New Oscillation Criteria for Second-Order Neutral Differential Equations with Delayed Arguments. Mathematics 2019, 7, 619. [CrossRef]

5. Bazighifan, O.; Elabbasy, E.M.; Moaaz, O. Oscillation of higher-order differential equations with distributed delay. J. Inequal. Appl. 2019, 2019, 55. [CrossRef]

6. Chatzarakis, G.E.; Elabbasy, E.M.; Bazighifan, O. An oscillation criterion in 4th-order neutral differential equations with a continuously distributed delay. Adv. Differ. Equ. 2019, 336, 1-9.

7. Cesarano, C.; Pinelas, S.; Al-Showaikh, F.; Bazighifan, O. Asymptotic Properties of Solutions of Fourth-Order Delay Differential Equations. Symmetry 2019, 11, 628. [CrossRef]

8. Cesarano, C.; Bazighifan, O. Oscillation of fourth-order functional differential equations with distributed delay. Axioms 2019, 8, 61. [CrossRef]

9. Cesarano, C.; Bazighifan, O. Qualitative behavior of solutions of second order differential equations. Symmetry 2019, 11, 777. [CrossRef]

10. Bazighifan, O.; Cesarano, C. A Philos-Type Oscillation Criteria for Fourth-Order Neutral Differential Equations. Symmetry 2020, 12, 379. [CrossRef]

11. El-Nabulsi, R.A.; Moaaz, O.; Bazighifan, O. New Results for Oscillatory Behavior of Fourth-Order Differential Equations. Symmetry 2020, 12, 136. [CrossRef]

12. Grace, S.; Lalli, B. Oscillation theorems for nth order nonlinear differential equations with deviating arguments. Proc. Am. Math. Soc. 1984, 90, 65-70. [CrossRef]

13. Grace, S.; Agarwal, R.; Graef, J. Oscillation theorems for fourth order functional differential equations. J. Appl. Math. Comput. 2009, 30, 75-88. [CrossRef]

14. Liu, S.; Zhang, Q.; Yu, Y. Oscillation of even-order half-linear functional differential equations with damping. Comput. Math. Appl. 2011, 61, 2191-2196. [CrossRef]

15. Li, T.; Baculikova, B.; Dzurina, J.; Zhang, C. Oscillation of fourth order neutral differential equations with p-Laplacian like operators. Bound. Value Probl. 2014, 56, 41-58. [CrossRef]

16. Kiguradze, I.T.; Chanturiya, T.A. Asymptotic Properties of Solutions of Nonautonomous Ordinary Differential Equations; Kluwer Academic Publishers: Dordrecht, The Netherlands, 1993.

17. Moaaz, O.; El-Nabulsi, R.A.; Bazighifan, O. Oscillatory Behavior of Fourth-Order Differential Equations with Neutral Delay. Symmetry 2020, 12, 371. [CrossRef]

18. Moaaz, O.; El-Nabulsi, R.A.; Bazighifan, O. Behavior of Non-Oscillatory Solutions of Fourth-Order Neutral Differential Equations. Symmetry 2020, 8, 479. [CrossRef]

19. Moaaz, O.; Elabbasy, E.M.; Bazighifan, O. On the asymptotic behavior of fourth-order functional differential equations. Adv. Differ. Equ. 2017, 2017, 261. [CrossRef]

20. Moaaz, O.; Elabbasy, E.M.; Muhib, A. Oscillation criteria for even-order neutral differential equations with distributed deviating arguments. Adv. Differ. Equ. 2019, 2019, 297. [CrossRef] 
21. Moaaz, O.; Awrejcewicz, J.; Bazighifan, O. A New Approach in the Study of Oscillation Criteria of Even-Order Neutral Differential Equations. Mathematics 2020, 8, 197. [CrossRef]

22. Park, C.; Moaaz, O.; Bazighifan, O. Oscillation Results for Higher Order Differential Equations. Axioms 2020, 9, 14. [CrossRef]

23. Zhang, C.; Agarwal, R.P.; Bohner, M.; Li, T. New results for oscillatory behavior of even-order half-linear delay differential equations. Appl. Math. Lett. 2013, 26, 179-183. [CrossRef]

24. Zhang, C.; Li, T.; Suna, B.; Thandapani, E. On the oscillation of higher-order half-linear delay differential equations. Appl. Math. Lett. 2011, 24, 1618-1621. [CrossRef]

25. Moaaz, O.; Dassios, I.; Bazighifan, O. Oscillation Criteria of Higher-order Neutral Differential Equations with Several Deviating Arguments. Mathematics 2020, 8, 412. [CrossRef]

26. Bazighifan, O. Kamenev-Type Asymptotic Criterion of Fourth-Order Delay Differential Equation. Fractal Fract 2020, 4, 7. [CrossRef]

27. Agarwal, R.P.; Grace, S.R.; O'Regan, D. Oscillation criteria for certain nth order differential equations with deviating arguments. J. Math. Appl. Anal. 2001, 262, 601-622. [CrossRef]

(c) 2020 by the authors. Licensee MDPI, Basel, Switzerland. This article is an open access article distributed under the terms and conditions of the Creative Commons Attribution (CC BY) license (http://creativecommons.org/licenses/by/4.0/). 